
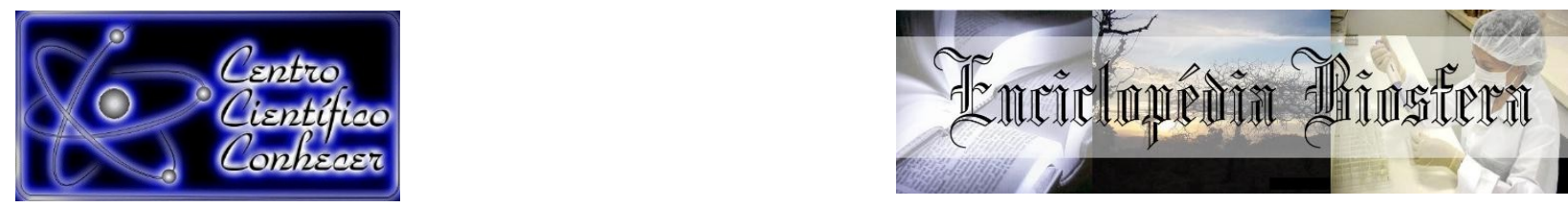

\title{
SUPORTE RESPIRATÓRIO EXTRACORPÓREO EM PACIENTES
}

Sarah Ribeiro de Oliveira ${ }^{1}$, Aldenira Matias de Moura ${ }^{1}$, Karen Ribeiro de Oliveira ${ }^{1}$, Nelite Rodrigues de Oliveira ${ }^{1}$, Murilo Barros Silveira ${ }^{2}$

1. Biomédica, Especialista em Perfusão (Circulação Extracorpórea), Instituto ASGARD, Goiânia, GO, Brasil.

2. Biomédico, Especialista em Microbiologia, Mestrando em Biologia da Relação Parasito-Hospedeiro (Imunoparasitologia), Instituto de Patologia Tropical e Saúde Pública, Goiânia, GO, Brasil. E-mail:murilo_bsilveira@hotmail.com

Recebido em: 15/02/2020 - Aprovado em: 15/03/2020 - Publicado em: 30/03/2020 DOI: 10.18677/EnciBio_2020A22

\begin{abstract}
RESUMO
O termo oxigenação por membrana extracorpórea (ECMO) foi inicialmente usado para descrever o suporte extracorporal a longo prazo que se concentrou sobre a função de oxigenação. O objetivo da terapia é para dar tempo para a recuperação intrínseca dos pulmões e do coração, assim uma circulação extracorpórea padrão fornece suporte durante vários tipos de procedimentos cirúrgicos cardíacos. 0 objetivo deste trabalho foi verificar os aspectos técnicos e clínicos sobre o uso de oxigenação por membrana extracorporal (ECMO). Para tanto, foi realizada pesquisa bibliográfica em diversas bases de dados (Bireme, Chest, Lilacs, PubMed e Scielo), além de Revista Intensiva, diversos livros, teses e textos eletrônicos onde foram buscados artigos nacionais ou internacionais, escritos por profissionais da saúde especializados no tema. Principalmente no caso de pacientes neonatos, a ECMO é indicada através dos seguintes aspectos: Recém-nascido com 34 semanas de idade, maior que 2,5kg ao nascer e sem coagulopatia. Em geral o paciente apoiado pela ECMO deve ser tratado como um paciente crítico, sendo que a analgesia pode ser administrada preemptivamente em todos os pacientes canulados, pelo menos até que seja possível uma avaliação objetiva da dor. Concluiu-se que não oferecer ECMO é como não oferecer a ventilação convencional, pois a ECMO é utilizada enquanto busca-se um diagnóstico e melhor estratégia terapêutica, dando ao paciente a chance de melhorar sua condição cardiorrespiratória aumentando as chances de recuperar a própria vida.
\end{abstract}

PALAVRAS-CHAVE: Disfunção cardiopulmonar; Ecmo; Sistema de oxigenação extracorpóreo.

\section{EXTRACORPOREAL RESPIRATORY SUPPORT IN PATIENTS}

\begin{abstract}
The term extracorporeal membrane oxygenation (ECMO) has been used to describe long-term extracorporeal support that focuses on oxygenation function. The goal of therapy is the intrinsic recovery time of the lungs and heart, as well as a standard cardiopulmonary bypass that is supported during various types of cardiac surgical procedures. The objective of this work was to verify the technical and clinical aspects about the use of extracorporeal membrane oxygenation (ECMO). For this purpose,
\end{abstract}


bibliographic research was carried out in several databases (Bireme, Chest, Lilacs, PubMed and Scielo), in addition to the Revista Intensiva, several books, theses and electronic texts where national or international articles were searched, written by medical professionals without theme. Especially in the case of neonatal patients, an ECMO is indicated by the following aspects: Newborn with 34 weeks of age, greater than $2.5 \mathrm{~kg}$ at birth and without coagulopathy. In general, the patient supported by ECMO should be treated as a critical patient, being an analgesia that can be administered preferentially in all cannulated patients, at least until an objective evaluation of the patient is possible. It was concluded that it does not offer ECMO as it does not offer conventional ventilation, since ECMO is used while seeking a diagnosis and a better therapeutic strategy, giving the patient a chance to improve his cardiorespiratory condition used as chances to recover a personal life.

\section{INTRODUÇÃO}

O termo ECMO (Oxigenação por membrana extracorpórea) é um método usado para descrever o suporte extracorpóreo a longo prazo concentrado na função de oxigenação corpórea, que posteriormente foi utilizado também no pós-operatório em pacientes submetidos em cirurgia cardíaca (LOWRY et al., 2013; SILVA, 2013; MASLACH-HUBBARD; BRATTON, 2014; DÍAZ et al., 2017). A ECMO tem se tornado uma grande ferramenta no arsenal de vida como suporte na circulação extracorpórea principalmente em adultos ou crianças com grave e refratária disfunção cardíaca ou pulmonar (LOWRY et al., 2013).

Com o avanço da tecnologia, diversos recursos foram testados e utilizados, uma nova modalidade foi estabelecida o suporte de vida extracorpórea (ECLS, 2017) que entrou em voga para descrever esta tecnologia (DÍAZ et al., 2017).

A história da ECMO inicia-se juntamente com história da circulação extracorpórea, quando, em 1953, Dr. Gibbon realizou a primeira cirurgia de coração aberto com sucesso utilizando a circulação extracorpórea. Em 1955, Richard DeWall desenvolveu o primeiro oxigenador de bolhas descartáveis, que apesar de inovador tem desvantagens sendo incompatíveis com suporte de longo prazo. Em 1957, Kammermeyer desenvolveu o polímero de dimetilpolisiloxano, conhecido como silicone, sendo um material altamente resistente à pressão hidrostática e muito eficaz na difusão de gás. Em 1963, Dr. Theodor Kolobow desenvolveu a primeira membrana de pulmão artificial (oxigenador de membrana de silicone), semelhante à utilizada atualmente. Em 1969 foi realizado o primeiro suporte prolongado bemsucedido com um oxigenador de membrana de silicone (SILVA, 2013; DÍAZ et al., 2017).

Em 1971, o Dr. JD Hill teve o primeiro caso com uso de um suporte extracorpóreo prolongado com sucesso, onde conectou a ECMO a um paciente de 24 anos com insuficiência respiratória devido à politraumatismo torácico, por 75 horas. Robert H. Bartlett, conhecido como o pai da ECMO, relatou, em 1972, a primeira ECMO cardíaca em uma criança após cirurgia cardíaca, e em 1975 primeiro ECMO neonatal realizado com sucesso, em um recém-nascido com insuficiência respiratória e hipertensão pulmonar secundária à aspiração de mecônio. Bartlett modificou a circulação extracorpórea para serem usadas nas unidades de terapia intensiva (GILBERTO; FILHO 2000; FORTUNATO JÚNIOR, 2010; DÍAZ et al., 2017; LIN, 2017; VAN OMMEN et al., 2018).

Após o relato bem-sucedido em 1975, houveram vários estudos que relataram uma sobrevida significativa de recém-nascidos submetidos à ECMO por insuficiência respiratória grave reversível por consequência de várias patologias, tornando a 
ECMO nessa faixa etária uma opção de tratamento padrão. Já em adultos não demostraram tanto sucesso, porém após a pandemia de H1N1 em 2009, houve um crescente uso da ECMO em adultos (GILBERTO; FILHO, 2000; MAKSOUD et al., 2001; DÍAZ et al., 2017; LIN, 2017; GATZWEILER et al., 2018).

Descrito na literatura, outro uso da ECMO é um estudo que demonstrou uma série de casos de jovens que tiveram a taxa de sobrevida aumentada após a cirurgia com diagnóstico de insuficiência respiratória irreversível e refratária à ventilação convencional que utilizaram a ECMO como ponte para transplante pulmonar (COSTA et al., 2011; GATZWELER et al., 2018).

No Brasil, o uso da ECMO está em ascensão na assistência circulatória mecânica, principalmente em hospitais com atendimentos de alta complexidade, o que demanda profissionais especializados para o cuidado do paciente (COSTA et al., 2011). Assim, objetivou se com o presente estudo, elaborar uma revisão de literatura abordando os aspectos do suporte respiratório extracorpóreo em pacientes e descrever os aspectos clínicos envolvidos.

\section{MÉTODOS}

O presente estudo é caracterizado como revisão, com o propósito de buscar informações relacionadas ao tema de estudo. O levantamento dos artigos foi realizado nas seguintes bases de dados: PubMed (Public Medical Literature Analysis and Retrieval System Online), Scielo (Scientific Eletronic Library Online) e Lilacs (Literatura Latino-Americana e do Caribe em Ciências da Saúde). Foram utilizados as seguintes palavras-chave: Circulação extracorpórea, oxigenação extracorpórea, Disfunção cardiopulmonar, reabilitação. A busca dos artigos foi executada no período de janeiro à novembro do ano de 2019. Os critérios de inclusão foram artigos em português e inglês, disponíveis na íntegra e que tivessem relação com o tema. Os critérios de exclusão foram artigos que não estivessem esclarecidos sobre a funcionalidade e os aspectos da ECMO.

\section{Circuito de ECMO}

O aparelho da ECMO é constituído por um cateter para drenagem venosa conectada a um tubo ligado a um reservatório, que passa por uma bomba de rolete, que impulsiona o sangue através de um oxigenador de membrana, depois o sangue é enviado a um termorregulador, e retorna ao paciente através do cateter arterial (Figura 1) (GILBERTO;FILHO, 2000) (MASLACH-HUBBARD; BRATTON, 2014).

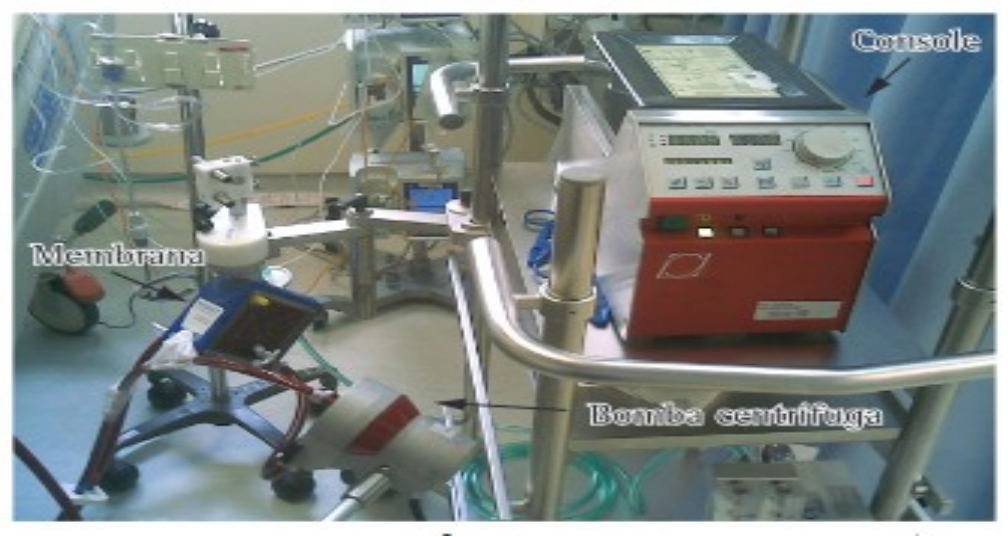

FIGURA 1. Sistema de Oxigenação Extracorpórea (Adaptado Azevedo et al., 2012). 
A maioria dos sistemas usava bombas de rolete (semi-oclusivas), porém atualmente $\mathrm{o}$ uso de bombas centrífugas tem aumentado pelas vantagens que oferece. Como a drenagem é feita por gravidade, no sistema com bombas de rolete é necessário um reservatório, assim aumentam o fluxo turbulento e a chance de ruptura da tubulação tornando necessário um circuito mais longo. Já as bombas centrífugas, devido aos acionamentos magnéticos, permitem o uso de tubos mais curtos e menores volumes de prime (GILBERTO;FILHO, 2000) (MASLACHHUBBARD; BRATTON, 2014)

Os oxigenadores de silício usados no início tinham superfície muito grande, que dificultava preparar e desintegrar e seu uso estava associada a uma grande resposta inflamatória. Foram desenvolvidos os oxigenadores de fibra oca de polipropileno, que pareciam provocar menos inflamação, porém tem uma facilidade para extravasar o plasma, fazendo com que o tempo de vida do oxigenador diminua. Atualmente, são usados oxigenadores de fibra oca não porosos, de polimetilpenteno, que fornecem troca de gases mais eficiente e baixa resistência ao fluxo, além de usarem menor volume de injeção (MASLACH-HUBBARD; BRATTON, 2014).

Através de um mecanismo do tipo contracorrente, o permutador de calor aquece o sangue. $O$ sangue é exposto à água quente que circula dentro de tubos de metal (MEADE et al., 2008; SILVA, 2013).

\section{Tipos de ECMO}

A ECMO possui duas configurações de canulações que dependem do tipo de suporte que o paciente necessita, podendo ser ECMO veno-venoso (VV) ou ECMO veno-arterial (VA) (Figura 2).

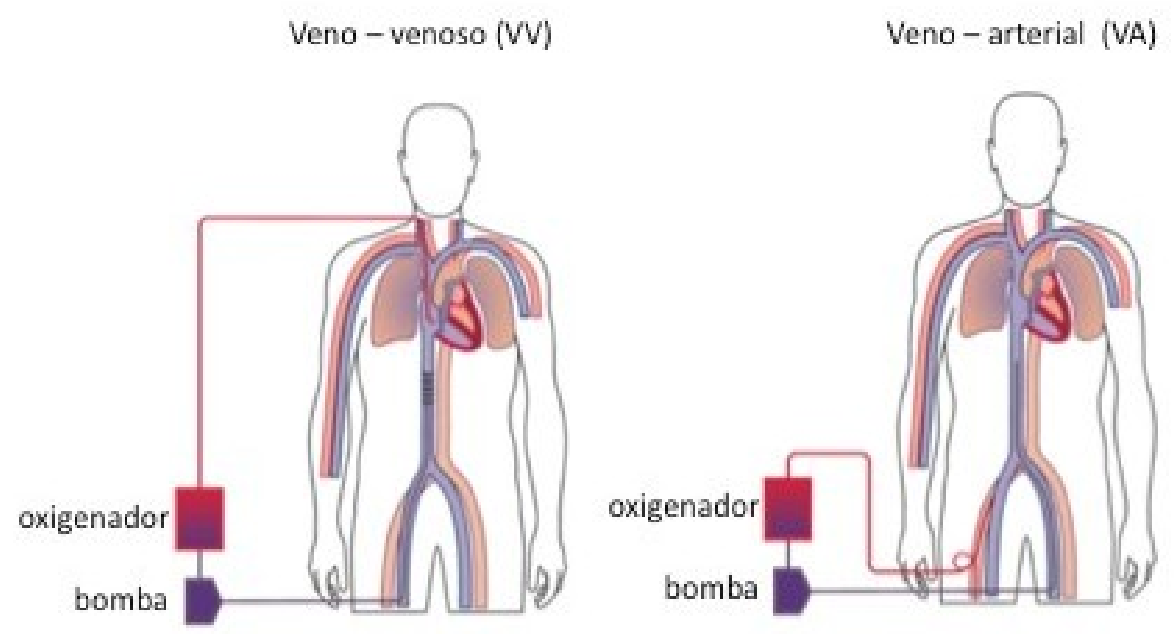

FIGURA 2. Sistema ECMO veno-venoso (VV) e ECMO veno-arterial (VA).

Na ECMO veno-venoso, é colocado uma cânula de duplo lúmen através da veia jugular direita para o átrio direito, o sangue drenado passa pelo circuito de ECMO, e retorna através do lúmen interno do cateter e é inclinado a dirigir o sangue através da válvula tricúspide. A ECMO VV é contra indicada em casos com insuficiência ventricular direita, hipertensão arterial pulmonar que requer ENCICLOPÉDIA BIOSFERA, Centro Cientifico Conhecer - Jandaia-GO, v.17 n.31; p. 2282020 
vasodilatadores pulmonares exógenos significativos, obstrução venosa pulmonar, e insuficiência ventricular esquerda ou qualquer condição clínica que limite o retorno venoso sistêmico do paciente. (GILBERTO; FILHO, 2000; SILVA, 2013; LIN, 2017; VAN OMMEN, 2018)

Na ECMO veno-arterial o sangue é drenado através de uma a cânula colocada da veia jugular direita para dentro do átrio direito, passa pelo circuito de ECMO, e retorna ao paciente através de uma cânula colocada na artéria carótida direita no arco aórtico. A configuração da ECMO VA suporta tanto a troca gasosa quanto suplementa o débito cardíaco (GILBERTO; FILHO, 2000; SILVA, 2013; LIN, 2017; VAN OMMEN, 2018)

Dependendo da indicação clínica, diferentes híbridos canulações podem ser usadas. As duas razões clássicas para usar uma configuração híbrida são melhorar a drenagem sanguínea (fornecer suporte circulatório) e evitar síndrome de Harlequin (hipoxemia restrita à parte superior do corpo) (GATZWELLER et al., 2018). Às vezes o fluxo sanguíneo através da veia não atende à demanda gerada pela bomba. Como resultado, a pressão negativa pode sugar a parede venosa para os orifícios de acesso da cânula, situação referida como fenômeno de "sucção", levando a obstrução transitória das entradas e uma diminuição no fluxo sanguíneo da ECMO. A obstrução é geralmente breve (às vezes frações de segundo) até a veia novamente ser preenchida com sangue proveniente do retorno venoso (WUNSCH et al., 2013; VILLA et al., 2015). O processo se repete indefinidamente e geralmente leva à vibração do circuito da ECMO. As correções imediatas envolvem a redução do sangue da ECMO fluxo (que pode piorar a hipoxemia) ou administrar desafios de fluidos (que podem piorar a função pulmonar) (SCHAIBLE et al., 2010; VAN OMMEN et al., 2018).

Dentre os maiores desafios para um programa eficiente de ECMO é treinar uma equipe de especialistas em ECMO, esse profissional é responsável pelo sucesso da ECMO. Esse profissional é responsável pelos cuidados contínuos com o circuito e com os controles de heparinização e trocas gasosas pela membrana. Em centros de ECMO, enfermeiros e perfusionistas podem se qualificar para a tarefa após treinamento adequado. Entretanto, os perfusionistas implicam em um custo adicional em exemplo aos hospitais, pois são profissionais especializados que habitualmente não estão à disposição de hospitais pediátricos que não realizam cirurgias cardíacas (MEADE et al., 2008; SCHAIBLE et al., 2010; PARK et al., 2011).

\section{Indicações para ECMO}

Os critérios para indicação de ECMO no caso de pacientes neonatos, é utilizado pela maioria dos centros de ECMO para seleção, são: recém-nascido (RN) 34 semanas de idade gestacional; maior que 2,5 kg ao nascer; sem coagulopatia; sem cardiopatia congênita; em ventilação mecânica por menos de 15 dias; não existir malformações letais; lesão pulmonar reversível. Além dos critérios para seleção são aplicados critérios de qualificação específicos como: índice de oxigenação >40; deterioração aguda do quadro clínico; parada ou falência cardíaca; barotrauma; gradiente alvéolo-arterial de oxigênio $>620 \mathrm{mmHg}$; ausência de resposta a todos os outros tratamentos disponíveis (GILBERTO; FILHO, 2000; MAKSOD, 2001; SILVA, 2013; KATTAN et al., 2017; GATZWEILER et al., 2018; VAN OMMEN et al., 2018).

Já os critérios para indicação de ECMO em pediatria são: baixo débito cardíaco resultante insuficiência da direita, esquerda e biventricular após reparação de defeito congênito; crise vaso-reativa pulmonar após reparo de defeito cardíaco congênito 
levando a hipoxemia grave, baixo débito cardíaco, ou ambos; como uma ponte para a cirurgia cardíaca em pacientes com grave lesão de órgão-final resultante do baixo débito cardíaco relacionado à doença cardíaca congênita; como uma ponte para o transplante cardíaco e, como uma ponte para a recuperação temporária na cardiomiopatia secundária a insuficiência renal, a miocardite, e queimaduras (SILVA, 2013).

\section{Cuidado com o Paciente}

$O$ paciente apoiado pela ECMO deve ser tratado como um paciente crítico. A analgesia pode ser administrada preemptivamente em todos os pacientes canulados, pelo menos até que seja possível uma avaliação objetiva da dor (CALLAWAY et al., 2015). A sedação deve ser titulada para promover ou facilitar a ventilação protetora ou ultraprotetora durante as primeiras horas de suporte à ECMO, sempre evitando risco de vida agitação (BINGHAM et al., 2018; GRANT et al., 2019).

Alguns pacientes precisam de bloqueadores neuromusculares para garantir a ventilação mecânica adequada nas primeiras horas do suporte à ECMO.

A alimentação deve ser iniciada o mais rápido possível, seu impacto no fluido equilíbrio nos primeiros dias de apoio à ECMO deve ser levado em consideração. Antimicrobianos não devem ser usados profilaticamente. Complicações infecciosas durante o suporte ECMO, principalmente a pneumonia associada a ventilação são comuns e devem ser tratadas como de costume (GRANT et al., 2018; GRANT et al., 2019).

\section{Contraindicações para ECMO}

O descumprimento dos critérios de seleção e indicações citados para oxigenação por membrana extracorpórea acima são uma contraindicação para o uso da ECMO, além de patologias irreversíveis e pré-existentes que prejudicam a qualidade de vida (CHANDRA et al., 2012; MARQUES et al., 2017). Porém, quando é considerado a ECMO em um paciente pediátrico, não existe um conjunto claro de critérios de inclusão ou exclusão, sendo assim, a avaliação é bastante fundamentada na situação do paciente e experiência institucional com ECMO pediátrica (GILBERTO; FILHO, 2000; SILVA, 2013; MARQUES, 2013).

\section{Complicações da ECMO}

As complicações referentes à ECMO podem ser divididas em dois tipos: mecânicas, que são decorrentes do circuito extracorpóreo, e clínicas, que são as que acontecem no paciente (BUNGE et al., 2018; GATZWELLER et al., 2018).

As complicações mecânicas, mais comum são coágulos no circuito, que podem acarretar a falha do oxigenador, coagulopatia de consumo, e embolias. $\mathrm{Na}$ colocação da cânula, pode causar danos na veia jugular, podendo ocasionar hemorragia massiva do mediastino, e lesão na artéria carótida pode gerar uma dissecção da aorta letal (DALTON; MACRAE, 2015). Falhas do circuito, como a fonte de oxigênio e misturadores de oxigênio, falha dos equipamentos de monitoramento, rachaduras nos conectores, ruptura de tubo, e mau funcionamento da bomba, podendo ser apenas um retorno venoso inadequado (GILBERTO; FILHO, 2000; SILVA, 2013).

As complicações clínicas normalmente são consequências da anticoagulação, das interações do sangue com as superfícies artificiais do circuito e das mudanças no padrão de fluxo sanguíneo. Complicações neurológicas, como convulsões e 
hemorragias intracranianas. Complicações hemorrágicas, como hemorragias intratorácica, abdominal ou retroperitoneal, hemorragia no local cirúrgico, no local da cânula, ou no local de um procedimento anterior, e contagem de plaquetas diminuídas (KATTAN et al., 2017; OUELLETTE, et al., 2017). Complicações cardíacas como atordoamento do miocárdio, além da hipertensão que aumenta o risco de hemorragia e acidente vascular cerebral. Complicação pulmonar, como pneumotórax e/ou hemorragia pulmonar. Complicação renal, como oligúria é a mais frequente, porém pode ocorrer necrose tubular aguda necessitando de hemofiltração e hemodiálise (GRAY et al., 2015; LAZZERI et al., 2019).

Complicações do trato gastrointestinal como hemorragia pode ocorrer como consequência de estresse, isquemia, ou tendências hemorrágicas, hiperbilirrubinemia direta e cálculos biliares ocasionados pelo jejum prolongado e nutrição parenteral (ESTEBAN et al., 2013). Complicações metabólicas incluem acidose ou alcalose; hipercalemia ou hipocalemia; hipernatremia ou hiponatremia; hipercalcemia ou hipocalcemia; hiperglicemia ou hipoglicemia; além da ECMO poder alterar a concentração sérica de drogas devido ao aumento do volume de distribuição. Complicações como infecção e sepse podem ocorrer, devido ao circuito apresentar como um corpo estranho (SILVA, 2013; DÍAZ, 2015; GONZÁLEZPARDO, 2016; KATTAN et al., 2017; LIN, 2017; GATZWELER et al., 2018).

A ECMO é um fator de risco adicional para o desenvolvimento de infecção. Os pacientes em ECMO comumente encontram-se em uso de múltiplos dispositivos invasivos, como cateter de artéria pulmonar, cateter para monitorização da pressão arterial invasiva e cateter venoso central, o que aumenta o risco de infecção de corrente sanguínea, sendo este diretamente proporcional ao tempo de permanência em ECMO (JAIN et al., 2009; LUBNOW et al., 2014; REEB et al., 2016).

O diagnóstico de infecção em paciente em ECMO pode ser difícil, visto que sinais clínicos e sintomas clássicos associados à infecção hospitalar, como febre e leucocitose, podem não estar presentes. O paciente pode ser incapaz de elevar a temperatura corpórea e cursar com febre, principalmente devido à perda de calor pelo circuito da ECMO. O paciente em ECMO frequentemente possui aumento dos leucócitos secundário à circulação extracorpórea, uma vez que o sangue circula pelo circuito não epitelizado da $\mathrm{ECMO}$, desencadeando resposta inflamatória. Devido à dificuldade em estabelecer o diagnóstico de infecção, muitos centros utilizam cultura de vigilância de rotina nos pacientes em ECMO (MOREIRA et al., 2018).

\section{Anticoagulação durante a ECMO}

A anticoagulação durante a ECMO é utilizada para prevenir complicações trombóticas, e tem sido muito estudada na tentativa de encontrar formas para aprimorar as técnicas de anticoagulação (MILTIADES et al., 2017; LO-PINTO et al., 2018). A heparina é mais frequentemente usada, e o monitoramento normalmente é feito através dos testes de tempo de coagulação ativada (TCA) e de tempo de tromboplastina parcial ativada (TTPa), de níveis de fibrinogênio, tempo de atividade da protrombina (TAP) e a contagem de plaquetas que são rotineiramente conferidos e corrigidos se necessário. O TCA é mantido entre 180 e 200 segundos, porém pode ser considerada a diminuição do TCA em situações com sangramento ativo (ROELEVELD, 2017; BINGHAM, 2018; VAN OMME et al., 2018).

\section{Aspectos Hematológicos}

Para otimizar o fornecimento de oxigênio, a hemoglobina do paciente deve ser mantida a 12-15 g / dL utilizando concentrado de hemácias. As cirurgias extensas 
como cirurgia cardíaca devido a necessidade acentuada de transfusões de hemácias induz níveis elevados de Hemoglobina, além disso, distúrbios metabólicos, hipóxia devido a parada cardíaca, várias doenças, sangramentos e outras grandes intervenções cirúrgicas podem aumentar as transfusões de hemácias com níveis aumentados de hemoglobina (MOK et al., 2016; PINSKY, 2016; ROELEVELD et al., 2016). Como resultado do consumo de plaquetas durante o ECMO, as transfusões de plaquetas são necessárias para manter a contagem de plaquetas acima de 100.000 / mcL. Tempo de coagulação ativado deve ser mantido a 180-240 segundos para evitar complicações hemorrágicas (GLADWIN et al., 2012; SCHOPKA et al., 2015).

A hemólise continua sendo uma complicação crítica durante o suporte à ECMO. Na literatura, a frequência de hemólise variou entre 5 e $18 \%$. A proporção de anormal altos níveis de hemoglobina na ECMO foram significativamente maiores para VA (4\%) em comparação com o tratamento com VMO ECMO (2\%). Além disso, a proporção de pacientes que apresentaram altos valores de hemoglobina durante a ECMO foi maior para o ECMO VA. Esses pacientes necessitaram de quantidades maiores de derivados do sangue (por um fator de 2,5 a mais de hemácias). Um estudo retrospectivo demonstrou que a transfusão de hemácias durante a ECMO foi associada a complicações graves (complicações tromboembólicas, sepse, hemólise) (MEHTA et al., 2015; STRUNINA et al., 2019).

\section{Aspectos Clínicos}

As complicações neurológicas incluem convulsões, hemorragias intracranianas e infarto podem ser devido à ligadura da artéria carótida e a veia jugular interna, heparinização sistêmica, trombocitopenia, coagulopatia ou hipertensão sistólica (ROCCO et al., 2004;THILLE et al., 2016; NOSOTTI et al., 2016).

As complicações hemorrágicas incluem hemorragias e contagem de plaquetas diminuídas. Podem ocorrer hemólise e coagulopatia de consumo. Hemorragia no local cirúrgico, no local da cânula, ou no local de um procedimento invasivo anterior, é uma complicação frequente da causa de heparinização sistêmica. Também podem ocorrer hemorragia intratorácica, abdominal ou retroperitoneal. Diminuições na contagem de plaquetas ocorrem devido à diminuição da produção, aumento do consumo, sequestro ou diluição (MAGGIORE et al., 2014; LYU et al., 2016).

Complicações cardíacas incluem atordoamento do miocárdio, as quais são definidas como uma diminuição na percentagem de encurtamento do ventrículo esquerdo, por mais de $25 \%$ com o início do ECMO que retorna ao normal após 48 horas de ECMO. Além disso, a hipertensão é uma complicação perigosa devido ao risco de hemorragia e acidente vascular cerebral. Arritmia pode ocorrer como resultado de hipoxia e de desequilíbrio eletrolítico (KHEMANI et al., 2015; LOU et al., 2015; LIN, 2017).

O pneumotórax é uma potencial complicação pulmonar, juntamente com hemorragia pulmonar. A oligúria é uma complicação renal frequentemente observada durante a primeira parte do ECMO; necrose tubular aguda é observada em alguns pacientes e pode exigir hemofiltração e hemodiálise. Complicações do trato GI (gastrointestinal) incluem hemorragia, que pode ocorrer como resultado de estresse, isquemia, ou tendências hemorrágicas. Hiperbilirrubinemia direta e cálculos biliares podem ocorrer secundários ao jejum prolongado e nutrição parenteral total (NPT), hemólise, e diuréticos. Complicações podem também resultar da infecção e sepse, porque o circuito de ECMO representa um corpo estranho 
grande intravascular, e a manipulação frequente aumenta o risco de sepse (KHILNANI et al., 2011; PARK et al., 2011)

Complicações metabólicas incluem: a) Acidose ou alcalose; b) Hipercalemia ou hipocalemia; c) Hipernatremia ou hiponatremia; d) Hipercalcemia ou hipocalcemia; e) Hiperglicemia ou hipoglicemia; f) O ECMO pode alterar a concentração sérica de drogas devido ao aumento do volume de distribuição. É necessária precaução quando as drogas terapêuticas estreitas são administradas, e as alterações de dose podem ser necessárias (PUTTER; SEGHATCHIAN, 2017).

Descompensação cardiorrespiratória aguda pode resultar devido a: a) Tamponamento cardíaco (a partir de sangue ou ar); b) Pneumotórax hipertensivo, ou hemotórax; c) Insuficiência respiratória; d) A isquemia miocárdica; e) Desequilíbrio eletrolítico; f) Hemorragia (hemorragia intracraniana, especialmente); g) Efeitos de drogas; h) Sepse esmagadora i) Período de desmame ou julgamento sem ECMO (PUTTER; SEGHATCHIAN, 2017).

Em pacientes com diagnóstico principal pré-ECMO de insuficiência respiratória, um período experimental sem ECMO está programado se: a) o paciente demonstra adequada troca gasosa em configurações ventilatórias razoáveis e; b) o paciente tolera a vazão da bomba de $10-20 \mathrm{ml} / \mathrm{kg} / \mathrm{min}$, com o mínimo de $200 \mathrm{~mL} / \mathrm{min}$. Tempo de desmame variáveis verificados em pacientes em ECMO. Além disso, a duração do tratamento varia (SU et al., 2010; TOUFEN et al., 2011).

\section{Uso de Fármacos}

Aminocapróico pode ser necessário para reduzir o sangramento durante a cirurgia. Somente sedação mínima sendo é necessária para a estabilização. As doses da maioria dos medicamentos inotrópicos, como a dopamina, dobutamina e epinefrina, geralmente podem ser reduzidas uma vez que o paciente está na oxigenação por membrana extracorpórea (ECMO). Diuréticos podem ser necessários para a mobilização de fluídos dos tecidos. Antiácidos e antagonistas $\mathrm{H}_{2}$ são geralmente administrados por hemorragia digestiva. Fenobarbital pode ser usado, se o paciente tem convulsões (CASSWELL et al., 2013; MUNSHI et al., 2019).

Antibióticos, tais como ampicilina e cefotaxima, são usados inicialmente nas dosagens típicas de septicemia; modificação de dosagem pode ser necessária, dependendo do agente patogênico e sensibilidade. A duração do tratamento varia de acordo com o diagnóstico inicial, e a avaliação diária do paciente. Deve-se lembrar que quanto mais tempo o paciente permanece nessa terapia, mais complicações o paciente pode experimentar (ZILBERG et al., 2008; SQUIERS et al., 2016; GRANT et al., 2018).

\section{CONSIDERAÇÕES FINAIS}

Visto a importância do uso da ECMO em recém-nascidos, é necessário que estudos sejam realizados em pacientes com indicação para a terapia, para que possamos nos aprofundar nos tratamentos, para aperfeiçoar técnicas e compreender a fisiopatologia de ECMO, com o propósito de reduzir complicações clínicas decorrentes do uso de ECMO. Além de necessários, mais estudos que comprovem a eficácia do tratamento, pois o ECMO nos dará tempo para pensar em diagnóstico e melhor estratégia terapêutica para o paciente, e tempo para recuperar da lesão pulmonar, e/ou lesão miocárdica que pode ser essencial para recuperar a vida. 


\section{REFERÊNCIAS}

AZEVEDO, L. C.; PARK, M.; COSTA, E. L.; SANTOS, E. V.; HIROTA, A. et al. Extracorporeal Support Study Group. Extracorporeal membrane oxygenation in severe hypoxemia: time for reappraisal? Journal of Brazilian Pneumology, v. 38, n. 1, p. 7-12, 2012.

BINGHAM, K. R.; RILEY, J. B.; SCHEARS, G. J. Anticoagulation Management during First Five Days of Infant-Pediatric Extracorporeal Life Support. The Journal of ExtraCorporeal Technology Colorado, v. 50, n. 9, p. 30-37, 2018. Disponivel em: https://www.ncbi.nlm.nih.gov/pmc/articles/PMC5848082/

BUNGE, J. J. H.; CALISKAN, K.; GOMMERS, D. Right ventricular dysfunction during acute respiratory distress syndrome and veno-venous extracorporeal membrane oxygenation. Journal Thoracic Disease, v. 10, n. 4, p. 674-682, 2018.

CALLAWAY, C. W.; DONNINO, M. W.; FINK, E. L. American Heart Association guidelines update for cardiopulmonary resuscitation and emergency cardiovascular care. Circulation, v. 132, n. 3, p. 465-482, 2015.

CASSWELL, G. K.; PILCHER, D. V.; MARTIN, R. S.; PELLEGRINO, V. A.; MARASCO, S. F. et al. Buying time: The use of extracorporeal membrane oxygenation as a bridge to lung transplantation in pediatric patients. Pediatric Transplant, v. 17, n. 8, p. 182-188, 2013.

CHANDRA, D.; STAMM, J. A.; TAYLOR, B.; RAMOS, R. M.; SATTEWHITE, L. KRISHAN, J. A. et a. Outcomes of noninvasive ventilation for acute exacerbations of chronic obstructive pulmonary disease in the United States, 1998-2008. Journal of Respiratory Critical Care Medicine, v. 185, n. 2, p.152-159, 2012.

COSTA, L. M. B.; HORA, M. P.; ARAUJO, E. O.; PEDREIRA, L. C. Cuidado de Enfermagem a uma paciente em uso da ECMO. Revista Baiana de Enfermagem, v. 25, n. 2 , p. $209-220,2011$.

DALTON, H. J.; MACRAE, D. J. Pediatric Acute Lung Injury Consensus Conference Group. Extracorporeal support in children with pediatric acute respiratory distress syndrome: proceedings from the Pediatric Acute Lung Injury Consensus Conference. Pediatric Critical Care Medicine, v. 16, n. 1, p. 111-117, 2015.

DA SILVA, V. D. ECMO - ASPECTOS CLíNICOS E TÉCNICOS. (2013). Disponível em: <https://ibrati.org/sei/docs/tese_701.doc>

DíAZ, R. G. Ecmo y ecmo mobile. soporte gardio respiratorio avanzado. Revista Médica Clínica Las Condes, v. 22, n. 3, p. 377-387, 2015. Disponível em: <https://doi.org/10.1016/s0716-8640(11)70438-2>

DÍAZ, R.; FAJARDO, C.; RUFS, J. Historia Del Ecmo (Oxigenación Por Membrana Extracorpórea O Soporte Vital Extracorpóreo). Revista Médica Clínica Las 
Condes, v. 28, n. 5, p. 796-802, 2017. Disponível em: https://doi.org/10.1016/j.rmclc.2017.10.004

\section{ECLS. Extracorporeal Life Support Organization. International ECLS Registry} Report, 2017.Disponível em: <https://www.elso.org>.

ESTEBAN, A.; FRUTOS-VIVAS, F.; MURIEL, A.; FERGUSON, N. D.; PENUELAS, $O$. et al. Evolution of mortality over time in patients receiving mechanical ventilation. Journal of Respiratory Critical Care Medicine, v. 188, n. 1, p. 220230, 2013.

FORTUNATO JÚNIOR, J. ; Circulação Extracorpórea Prolongada Com Oxigenador De Membrana (Ecmo) Para Suporte De Pacientes Com Insuficiência Cardiopulmonar Aguda Refratária, 2010. Disponível em: <http://dspace.c3sl.ufpr.br/dspace/bitstream/handle/1884/24783/Tese_DR_Jeronimo. pdf;jsessionid=8D122ACECA0956F634B4F7F995164A48? sequence=1>

GATZWEILER, E.; HOPPE, B.; DEWALD, O.; BERG, C.; MULLER, A. et al. Extracorporeal membrane oxygenation support in a newborn with lower urinary tract obstruction and pulmonary hypoplasia: A case report. Journal of Medical Case Reports, v. 12, n. 1, p. 4-7, 2018. Disponível em: https://doi.org/10.1186/s13256018-1749-1

GILBERTO, J., \& FILHO, M. (2000). ECMO (oxigenação extracórporea por membrana). RBM Revista Brasileira de Medicina, (7), 1-11. Disponível em: http:// www. moreirajr. com. br/revistas. asp.

GLADWIN, M. T.; KANIAS, T.; KIM-SHAPIRO, D. B. Hemolysis and cell-free hemoglobin drive an intrinsic mechanism for human disease. The Journal of clinical investigation, v. 122, n. 4, p. 1205-1208, 2012. https://doi.org/ 10.1172/JCI62972

GONZÁLEZ-PARDO, O. M.; CASSALETT-BUSTILLO, G.; NÚÑEZ, F. ECMO respiratorio como opción terapéutica en falla respiratoria refractaria en la edad pediátrica. Revista Colombiana de Cardiologia, v. 23, n. 6,, p. 560-571, 2016. https://doi.org/10.1016/j.rccar.2016.01.021

GRANT, A. A.; HART, V. J.; LINEEN, E. B.; LAI, C.; GINZBURG E. The impact of na advanced ECMO program on traumatically injured patients: ECMO program on traumatically injured patients. Artificial Organs, v. 42, n. 11, p. 1043-1051, 2018.

GRANT, A. A.; LINEEN, E. B.; KLIMA, A.; VIANNA, R.; LOEBE, M.; GHODSIZAD, A. et al. Refractory traumatic bronchopleural fistula: Is extracorporeal membrane oxygenation the new gold standard? Journal of Cardiotoracic and Surgery, v. 35, n. 1 , p. $242-245,2019$. doi:10.1111/jocs. 14298

GRAY, B. W.; HAFT, J. W.; HIRSCH, J. C.; ANNICH, G. M.; HIRSCHL, R. B. et al. Extracorporeal life support. Experience with 2,000 patients. ASAIO journal, v. 61, n.1, p. 2-7, 2015. https://doi.org/10.1097/MAT. 0000000000000150

JAIN, S.; KAMIMOTO, L.; BRAMLEY, A. M.; SCHMITZ, A. M.; LOUIE, J. et al. Pandemic Influenza A (H1N1) Virus Hospitalizations Investigation Team. 
Hospitalized patients with 2009 H1N1 influenza in the United States, April-June 2009. Journal England of Medicine, v. 361, n. 20, p. 1935-1944, 2009.

KATTAN, J.; GONZÁLEZ, Á.; CASTILLO, A.; CANEO, L. F. Neonatal and pediatric extracorporeal membrane oxygenation in developing Latin American countries. Jornal de Pediatria, v. 93, n. 2, p. 120-129, 2017. Disponível em: https://doi.org/10.1016/j.jpedp.2017.01.002

KHEMANI, R. G.; SMITH, L. S.; ZIMMERMAN, J. J.; ERICKSON, S. Pediatric Acute Lung Injury Consensus Conference Group. Pediatric acute respiratory distress syndrome: definition, incidence, and epidemiology: proceedings from the Pediatric Acute Lung Injury Consensus Conference. Pediatria Critical Care Medicine, v. 16, n. 1, p. 23-40, 2015.

KHILNANI, G. C.; GALLE, A. D.; HADDA, V. Non-invasive ventilation after extubation in patients with chronic obstructive airways disease: a randomised controlled trial. Anaesth Intensive Care, v. 39, n. 3, p. 217-223, 2011.

LAZZERI, C.; BONIZZOLI, M.; CIANCHI, G. Right ventricular hypertrophy in refractory acute respiratory distress syndrome treated with venovenous extracorporeal membrane oxygenation support. Journal Cardiothoracic and Vascular Anesthesia, 2019. https://doi.org/10.1053/j.jvca.2019.08.035

LEHLE, K.; PHILLIP, A.; MULLER, T.; SCHETLLER, F.; BEIN, T.; SCHMID, C. et al. Flow dynamics of different adult ECMO systems: a clinical evaluation. Artificial organs, v. 38, n. 5, p. 380-398, 2014. https://doi.org/10.1111/ aor.12180

LIN, J. C.Extracorporeal Membrane Oxygenation for Severe Pediatric Respiratory Failure. Respiratory Care, v. 62, n. 6, p. 732-750, 2017. Disponível em: https://doi.org/10.4187/respcare.05338

LOU, S.; MACLAREN, G.; PAUL, E.; BEST, D.; DELZOPPO, C. et al. Hemo filtration is not associated with increased mortality in children receiving extracorporeal membrane oxygenation. Pediatric Critical Care Medicine, v. 16, n. 2, p. 161-166, 2015.

LYU, L.; LONG, C.; HEI F.; JI, B.; LIU, J.; et al. Plasma free hemoglobin is a predictor of acute renal failure during adult venous-arterial extracorporeal membrane oxygenation support. Journal of Cardiothoracic Vascular Anesthesia, v. 30, n. 4, p. 891-895, 2016.

LOWRY, A. W.; MORALES, D. L.; GRAVES, D. E.; KNUDSON, J. D.; SHAMSZAD, P.; KNUDSON, J. D. et al. Characterization of Extracorporeal Membrane Oxygenation for Pediatric Cardiac Arrest in the United States: Analysis of the Kids. Database.Pediatric and Cardiology, 2013.

LO-PINTO, H.; ALLYN, J.; PERSICHINI, R.; BOUCHET, B.; BROCHIER, G.; MARTINET, O. et al. Predictors of red blood cell transfusion and its association with prognosis in patients undergoing extracorporeal membrane oxygenation. The 
International journal of artificial organs, v. 41, n. 10, p. 644-652, 2018. https://doi.org/10.1177/ 0391398818785132

LUBNOW, M.; PHILIPP, A.; FOLTAN, M.; ENGER, T. B.; LUNZ, D. et al. Technical complications during veno-venous extracorporeal membrane oxygenation and their relevance predicting a system-exchange--retrospective analysis of 265 cases. PloS One, v. 9, n. 12, p. 23-31, 2014.

MAGGIORE, S. M.; IDONE, F. A.; VASCHETO, R. HADDA, V.; GALLE, A. D. et al. Nasal high-flow versus Venturi mask oxygen therapy after extubation. Effects on oxygenation, comfort, and clinical outcome. Journal of Respiratory Critical Care Medicine, v. 1, n. 190, p. 282-288, 2014.

MAKSOUD-FILHO, J. G. DINIZ, E. M. A.; CECCON, M. E. J.; GALVANI, A. N. L. D'ADREA, M.; PINHO, L.; VAZ, F. A. C. Circulação extracorpórea por membrana ( ECMO ) em recém-nascido com insuficiência respiratória por síndrome de aspiração meconial : efeitos da administração de surfactante exógeno Extracorporeal membrane oxygenation ( ECMO ) in a neonate with respiratory. Jornal de Pediatria, V. 77, n. 2, p. 243-248, 2001. Disponível em: http://dx.doi.org/10.1590/S002175572001000300016

MASLACH-HUBBARD, A. \& BRATTON, S. L. Extracorporeal membrane oxygenation for pediatric respiratory failure: History, development and current status. World Journal of Critical Care Medicine v. 2, n. 4, p. 20-29, 2014. Disponível em: https://doi.org/10.5492/wjccm.v2.i4.29xMARQUES, R.; PEREIRA, S.; ABECASIS, F.; VALENTE, S.; NEVES, J. P. et al. Uso de oxigenação extra corporal ( ECMO ) em recém-nascidos com hérnia diafragmática congénita - dois casos de sucesso. Acta Pediátrica Portuguesa, v. 44, n. 4, p. 172-175, 2017. Disponível em: < https://pjp.spp.pt//article/view/2687/2673 >

MEADE, M. O.; COOK, D. J.; GUYATT, G. H.; SLUTSKY, A. S.; ARABI, Y. M. et al. Ventilation strategy using low tidal volumes, recruitment maneuvers, and high positive end-expiratory pressure for acute lung injury and acute respiratory distress syndrome: a randomized controlled trial. JAMA, v. 299, n. 6, p. 637-645, 2008.

MEHTA, A. B.; SYEDA, S. N.; WIENER, R. S; WALKEY, A. J. Epidemiological trends in invasive mechanical ventilation in the United States: a population-based study. Journal of Critical Care, v. 30, n. 4, p. 1217-1221, 2015.

MILTIADES, A. N.; GERSHEGORN, H. B.; HUA, M. Cumulative Probability and Time to Reintubation in United States Intensive Care Units. Critical Care Medicine, v. 45, n. 2, p. 835-842, 2017.

MOK, Y. H.; LEE, J. H.; CHEIFETZ, I. M. Neonatal extracorporeal membrane oxygenation: update on management strategies and long-term outcomes. Neonatal Care, v. 16, n. 1, p. 26-36, 2016.

MOREIRA, F. T.; PALOMBA, H.; CHAVES, R. C.; BOUMAN, C.; SCHULTZ, M. J.; SERPA, N. A. Early versus delayed initiation of renal replacement therapy for acute kidney injury: an updated systematic review, meta-analysis, metaregression and trial 
sequential analysis of randomized controlled trials. Revista Brasileira de Terapia Intensiva, v. 30, n. 3, p. 376-384, 2018.

MUNSHI, L.; WALKEY, A.; GOLIGHER, E. Venovenous extracorporeal membrane oxygenation for acute respiratory distress syndrome: a systematic review and metaanalysis. Lancet Respiratory Medicine, v. 7, n. 3, p. 163-172, 2019.

NOSOTTI, M.; ROSSO, L.; TOSI, D.; PALLESCHI, A.; MENDOGNI, P. et al. Extracorporeal membrane oxygenation with spontaneous breathing as a bridge to lung transplantation. Interact Cardiovascular and Thoracic Surgery, v. 16, n. 1, p. 55-59, 2016.

OUELLETTE, D. R.; PATEL, S.; GIRARD, T. D. Liberation from mechanical ventilation in critically ill adults: an official american college of chest physicians/american thoracic society clinical practice guideline: inspiratory pressure augmentation during spontaneous breathing trials, protocols minimizing sedation, and noninvasive ventilation immediately after extubation. Chest, v. 1, n. 2, p. 140$151,2017$.

PARK, M.; COSTA, E. L.; AFONSO, J. J.; SAMANO, M. N.; CARVALHO, C. R. Extracorporeal membrane oxygenation as a bridge to pulmonary transplantation in Brazil: are we ready to embark upon this new age? Clinics, v. 66, n. 9, p. 1659-1661, 2011.

PINSKY, M. \& RISKY, M. R. The right ventricle: interaction with the pulmonary circulation. Critical Care, v. 20, n. 2, p. 20-29, 2016.

PUTTER, J. S.; SEGHATCHIAN, J. Cumulative erythrocyte damage in blood storage and relevance to massive transfusions. Selective insights into serial morphological and biochemical findings. Blood transfusion, v. 15, n. 4, p. 348-356, 2017. https://doi.org/10.2450/2017.0312-16

REEB, J.; OLLAND, A.; RENAUD, S.; LEJAY, A.; SANTELMO, N.; MASSARD, G. et al. Vascular access for extracorporeal life support: tips and tricks. Journal of Thoracic Disease, v. 8, n. 4, p. 353-363, 2016.

ROCCO, M.; DELL'ULTRI, D.; MORELLI, A.; SPADETTA, G.; CONTI, G. et al. Noninvasive ventilation by helmet or face mask in immunocompromised patients: a case-control study. Chest, v. 126, n.2, p. 1508-1515, 2004.

ROELEVELD, P. P. What is new in pediatric ECMO? 2016, a year in review.

European Journal of Heart Failure, v. 19, n. 2, p. 92-96, 2017. Disponível em: <https://doi.org/10.1002/ejhf.851>

SCHOPKA, S.; PHILLIP, A.; HILKER, M.; MULLER, T.; ZIMMERMAN, M. et al. Clinical course and long-term outcome following venoarterial extracorporeal life support-facilitated interhospital transfer of patients with circulatory failure. $\begin{array}{lllllll}\text { Resuscitation, } & \text { v. } & 2, & \text { n. } & 93, & \text { p. } & 53-5,\end{array}$ https://doi.org/10.1016/j.resuscitation.2015.05. 021 
SCHAIBLE, T.; HERMLE, D.; LOERSCH, F.; DEMIRAKCA, S.; REINSHAGEN, K. et al. A 20-year experience on neonatal extracorporeal mem-brane oxygenation in a referral center. Intensive Care Medicine, v. 36, n. 7, p.1229-1234, 2010.

SQUIERS, J. J.; LIMA, B.; DIMAIO, J. M. Contemporary extracorporeal membrane oxygenation therapy in adults: fundamental principles and systematic review of the evidence. Journal Thoracic and Cardiovascular Surgery, v. 152, n. 1, p. 20-32, 2016.

STRUNINA, S.; HOZMAN, J.; OSTADAL, P. The peripheral cannulas in extracorporeal life support. Biomedical Engineering, v. 64, n. 2, 127-133, 2019. https://doi.org/10.1515/bmt-2017-0107

SU, C. L.; CHIANG, L. L.; YANG, S. H.; CHANT, K.; KEPT, K. et al. Preventive use of noninvasive ventilation after extubation: a prospective, multicenter randomized controlled trial. Respiratory Care, v. 2, n. 57, p. 204-210, 2010.

THILLE, A. W.; BOISSIER, F.; BEM-GHEZALA, H.. Easily identified atrisk patients for extubation failure may benefit from noninvasive ventilation: a prospective before-after study. Critical Care, v. 1, n. 4, p. 20-32, 2016.

TOUFEN, C. Jr.; COSTA, E. L.; HIROTA, A. S.; LI, H. Y.; AMATO, M. B.; CARVALHO, C. R. Follow-up after acute respiratory distress syndrome caused by influenza a (H1N1) virus infection. Clinics, v. 66, n. 6, p. 933-937, 2011.

VAN OMMEN, C. H.; NEUNERT, C. E.; CHITLUR, M. B. Neonatal ECMO. Frontiers in Medicine, v. 5 , n. 2, p. 1-8, 2018. Disponível em: https://doi.org/10.3389/fmed.2018.00289

VILLA, G.; KATZ, N.; RONCO, C. Extracorporeal membrane oxygenation and the kidney. Cardiorenal Medicine, v. 6, n. 1, p. 50-60, 2015.

WUNSCH, H.; WAGNER, J.; HERLIM, M. ICU occupancy and mechanical ventilator use in the United States. Critical Care Medicine, v. 41, n. 2, p. 2712-2719, 2013.

ZILBERG, M. D.; LUIPPOLD, M. D.; SULSKY S. SHORR, A. F. Prolonged acute mechanical ventilation, hospital resource utilization, and mortality in the United States. Critical Care Medicine, v. 36, n. 4, p. 724-730, 2008. 